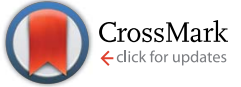

Cite this: DOI: 10.1039/c5ay02657a
Received 7th October 2015

Accepted 6th November 2015

DOI: 10.1039/c5ay02657a

www.rsc.org/methods

\section{Detection of prohibited treatment products on racing tires using headspace solid phase microextraction (SPME) and gas chromatography/ mass spectrometry (GC/MS)}

\begin{abstract}
W. D. Kranz, C. J. Carroll and J. V. Goodpaster*
A variety of commercial tire treatments are available that purport to help automobile tires better cling to the surface of a road or racetrack, raising concerns in the professional racing community that such products might be used to illicitly boost performance in competitive events. These tire treatments are reputed to cut lap times and improve handling and maneuverability. In some cases, the manufacturers even boast that their products are "undetectable" (i.e., impervious to the scrutiny of laboratory testing). In this study, a number of banned tire treatment products were evaluated principally by gas chromatography-mass spectrometry (GC-MS) using solid phase microextraction (SPME) as a pre-concentration technique. The chemicals off-gassed by each product were determined and grouped into two broad categories: 'plasticizer-based' tire treatment products and 'hydrocarbon-based' tire treatment products. This information was then applied to the analysis of genuine tire samples provided by the United States Auto Club (USAC), a professional racing association. Over the course of one year, 10 out of the 71 questioned samples tested positive for a prohibited treatment product. The manufacturers' claims regarding their products' invisibility to lab tests were largely proven to be unfounded: both the products themselves and the tires treated with them can be identified by a number of characteristic volatile compounds. These included known plasticizers such as pentanedioic acid diethyl ester, plasticizer-related compounds such as 2-ethyl-1-hexanol, and dearomatized distillates.
\end{abstract}

\section{Introduction}

Concerns about cheating in professional automobile racing circuits have been exacerbated by the introduction of a number of commercial products, which claim to have the ability to illicitly boost performance whilst avoiding traditional means of detection. Commercial tire treatments, in particular, have risen to become a major concern for the administrative bodies that regulate motorsports. These formulations are typically applied to the interior and/or exterior surfaces of the tires in the days and weeks leading up to a race. Over the intervening period of time, they absorb into the rubber, purportedly altering it physically and chemically in such a way as to augment the tires' grip on the surface of the racetrack. This, according to the vendors, translates to an improvement in handling, offering a subtle and subversive way to quicken lap times.

Although presently there are only a few commercial entities that specialize in this market, their catalogues are extensive. Several of the available tire treatments come stamped with the

Department of Chemistry and Chemical Biology, Forensic and Investigative Sciences Program, Indiana University Purdue University Indianapolis (IUPUI), 402 North Blackford Street, LD 326, Indianapolis, IN 46202, USA. E-mail: jvgoodpa@iupui.edu guarantee that they are "absolutely undetectable and odorless", specially-engineered to foil any attempt at conclusive analysis. For obvious reasons, the companies that produce them tend to be highly secretive about their formulations, reluctant to provide materials safety data sheets or to disclose any information whatsoever about their proprietary mixtures. More problematic still, published literature on tire treatments is virtually nonexistent, posing an informational challenge for the aspiring analyst.

The goals of this paper, then, are twofold: to offer insight into the yet-unreported nature of the existing tire treatment products, and to describe an effective method for the analysis of questioned tire samples.

Experiments were accomplished using gas chromatographymass spectrometry (GC-MS), with added sensitivity conferred by solid phase microextraction (SPME). SPME is a sample delivery technique that takes the place of liquid injection in the GC-MS process. When used in headspace mode, a coated fiber is exposed for some amount of time to the heated headspace above a sample of interest. After the volatile organic compounds off-gassed by the sample are adsorbed and pre-concentrated onto the fiber coating, the fiber is transferred to the heated injection port of the GC, where desorption of analyte molecules 
takes place. ${ }^{1-3}$ The simplicity and responsiveness of SPME make it well-suited to the ultrasensitive assay of nearly any conventional sample, and it proved especially useful during the course of this study, where the low volatility of the tire rubber provided a clean background for the highly-volatile tire treatment chemicals to propagate. Fourier transform infrared spectroscopy (FTIR) was also employed for the validation of GC-MS results, with the identities of the principal ingredients in every tire treatment confirmed wherever possible via ATR-FTIR.

The information provided here fills an important niche that has hitherto been vacant. For example, several reports describe the use of various methods, including SPME, for the analysis of volatile compounds emitted from tire rubber., ${ }^{4,5}$ The overwhelming interest in this area, however, are the volatiles emitted by rubber tires as they are manufactured, used and reclaimed. ${ }^{6,7}$ Similarly, SPME is widely recognized as a method to extract volatile compounds from the headspace above ignitable liquids ${ }^{8,9}$ and plasticized explosives. ${ }^{10-12}$ As will be discussed below, these compounds can be found in several tire treatment products discussed in this paper. There are several alternative methods for sampling volatiles emitted by a sample, including simple headspace, passive adsorption elution, and dynamic headspace concentration. SPME was selected in this case as it offers very high sensitivity, requires no solvents and is readily adapted to immediate analysis via GC/MS.

To the best of our knowledge, there are no peer-reviewed publications on the detection and analysis of liquid tire treatments on rubber automotive tires. Therefore, we believe this to be the first report on this topic. It is our hope that these findings will provide guidance to analysts who seek to evaluate questioned tire samples in the future, and that it will pave the way to a greater understanding of illicit commercial tire treatments.

\section{Materials and methods}

All tire treatment products and questioned tire samples were provided courtesy of the United States Auto Club.

A 6890 gas chromatograph coupled to a 5975 mass spectrometer (Agilent, Santa Clara, CA, USA) served as the principal instrumentation, with autosampler functionality provided by an MPS2 (Gerstel, Mülheim an der Ruhr, Germany). The column was a J\&W DB-5 ms $(30 \mathrm{~m} \times 0.25 \mathrm{~mm} \times 0.25 \mu \mathrm{m})$. The carrier gas was hydrogen, with a constant flow of $2.5 \mathrm{~mL} \mathrm{~min}^{-1}$. The oven temperature program utilized an initial temperature of $40{ }^{\circ} \mathrm{C}$ held for $1 \mathrm{~min}$, a ramp of $20{ }^{\circ} \mathrm{C} \mathrm{min}^{-1}$, and a final temperature of $300{ }^{\circ} \mathrm{C}$ held for $1 \mathrm{~min}$. The inlet temperature was $270{ }^{\circ} \mathrm{C}$. The mass transfer line temperature was $280{ }^{\circ} \mathrm{C}$. The mass spectrometer utilized an electron ionization detector at $230{ }^{\circ} \mathrm{C}$, with a scan range of $\mathrm{m} / \mathrm{z} 50-550$.

Tires were sampled by cutting out a piece of rubber with a mass of $\sim 1$ gram. The sample was then sealed within a $20 \mathrm{~mL}$ headspace vial and heated. Following a 5 min incubation time, a polydimethylsiloxane (PDMS) SPME fiber was inserted into the vial for $5 \mathrm{~min}$. All control and suspect tires were analyzed using a 5 min extraction time in splitless mode. The liquid tire treatment products were analyzed using a 3 min extraction time and a $32: 1$ split ratio.
The sensitivity of the method was gauged by conducting a serial dilution of Tire Treatment $\mathrm{B}$ and Tire Treatment $\mathrm{J}$ in pentane, then spiking $50 \mu \mathrm{L}$ of the diluted $10 \%, 1 \%$, and $0.1 \%$ solutions onto $1.0 \mathrm{~g}$ blank tire rubber cuttings and analyzing them using the same instrumental and SPME parameters. Based on the results of these analyses, a three point calibration curve of signal-to-noise ratio $(\mathrm{S} / \mathrm{N})$ versus concentration could be constructed, and the effective detection limits for various peaks of interest were calculated using the slope of the calibration curve and the standard error of the $y$-intercept.

Fourier transform infrared spectroscopy (FTIR) analysis of the tire treatment products was also completed. The instrumentation was a SpectrumOne (PerkinElmer, Waltham, MA, USA) equipped with a universal attenuated total reflectance (ATR) accessory. A drop of each tire treatment product was placed on the diamond crystal and allowed to evaporate, leaving only a thin film. Spectra were collected in absorbance mode. Signal averaging was performed over 16 scans with a scan resolution of $4 \mathrm{~cm}^{-1}$.

\section{Results and discussion}

\section{Analysis of tire treatment products}

Table 1 summarizes the results of the GC-MS analysis (Fig. 1) and the FTIR analysis (Fig. 2). Wherever possible, the major "red flag" peaks were identified, and an infrared library match was reported.

The tire treatment products can be grouped into two broad classes: products whose headspace is composed of a simple, two-to-four-component mixture of plasticizer-related compounds, which we define as the "plasticizer-based" class; and products that give off a complex suite of chemicals, largely normal and branched alkanes, cycloalkanes, and alkyl benzenes, which we define as the "hydrocarbon-based" class. These classes have been separated on Fig. 1 and 2 to highlight the intrinsic differences between them.

Characteristic compounds for the plasticizer-based tire treatments include butane-, pentane-, and hexanedioic acid diethyl ester. These bear structural similarity to a number of widely-used plasticizers, such as dioctyl adipate and bis(2-ethylhexyl) adipate. 2-Ethyl-1-hexanol also registers among the most telltale compounds, and is a known hydrolysis product of the plasticizers bis(2-ethylhexyl) phthalate, bis(2-ethylhexyl) adipate, bis(2-ethylhexyl) sebacate..$^{13-16}$ In a number of products, the 2-ethylhexyl moiety is present in the form of benzoic acid 2ethylhexyl ester, suggesting a possible source of origin.

The hydrocarbon-based products are more difficult to characterize, for they run the gamut of simple organic molecules, from normal and branched alkanes to alkenes, mono-, di-, tri-, and tert-substituted cycloalkanes, alkylbenzenes, and naphthenics. There appears to be no uniformity among them, aside from their general aspect as an unresolved envelope of peaks. In some formulations, the aliphatics and aromatics are accompanied by a selection of plasticizer-related compounds, such as pentanedioic acid diethyl ester and benzoic acid 2-ethylhexyl ester in Tire Treatment $\mathrm{H}$. 
Table 1 Table of all commercial tire treatments evaluated in this study, with the major components identified via GC-MS and FTIR highlighted. FTIR match scores are reported in parenthesis

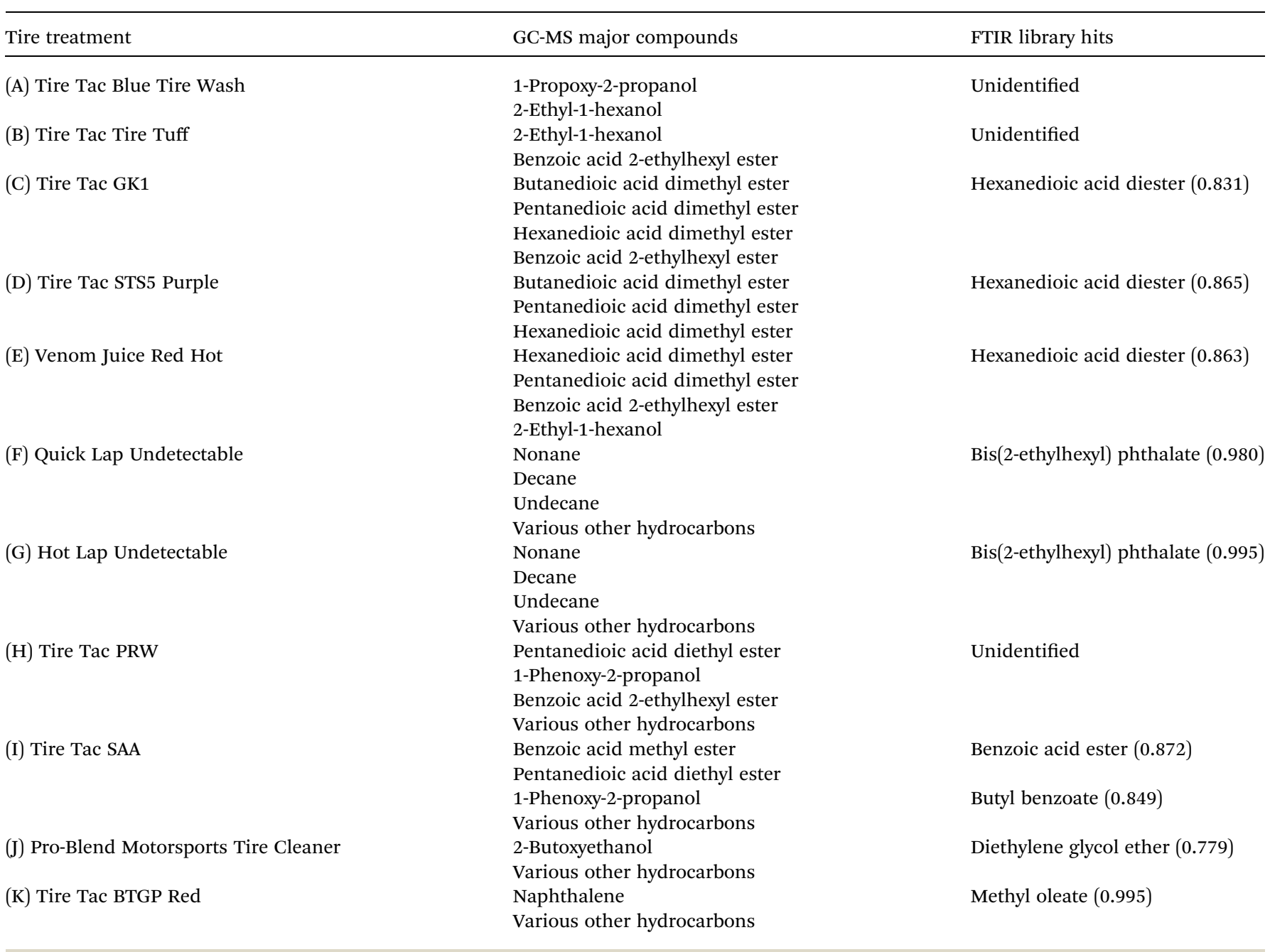

FTIR analysis largely served to confirm the primary ingredients in each tire treatment, although not all treatments could be successfully characterized. The chemical differences between the different classes are manifest in the infrared spectra, with those falling under the plasticizer-based umbrella yielding a relatively simple fingerprint region, and those in the hydrocarbon-based category proving more complex. Tire Treatments A and $\mathrm{J}$ were aqueous-based, as indicated by the hydroxyl peak present in both infrared spectra and the solvents' general reluctance to evaporate, while the rest were all purely organic.

\section{Sensitivity study}

The results of the serial dilution study showed the method was quite sensitive to the presence of illicit tire treatments. Tire Treatment B was an arbitrarily-chosen exemplar from the plasticizer-based class, while Tire Treatment J was an arbitrarily-chosen exemplar from the hydrocarbon-based class. Calibration curves indicated that butanedioic acid diethyl ester could be detected in tires spiked with Tire Treatment B at effective amounts as low as $200 \mathrm{~nL}$ tire treatment per gram of tire rubber, while pentanedioic acid diethyl ester could be detected at $500 \mathrm{~nL} \mathrm{~g}^{-1}$. The limits of detection for Tire Treatment J were similar, with nonane, decane, and undecane each detectable at $200 \mathrm{~nL} \mathrm{~g}^{-1}$. Each compound's limit of quantitation is $\sim 3.3$ times higher than its respective detection limit, giving an estimated lower limit of quantitation of $600-1600 \mathrm{~nL} \mathrm{~g}^{-1}$.

\section{Analysis of questioned tire samples}

Over the course of one year, over seventy tire samples were submitted for analysis. In that time, ten tires returned a positive result for the presence of illicit tire treatments. We present three case studies highlighting the analysis of these tires and the conclusions that were drawn.

The results of the first case study are depicted in Fig. 3. Six questioned tires were submitted for analysis along with a "track tire"- a tire provided by USAC that was run on the same racetrack and race day as the other exemplars, which functioned as a negative control. The track tire bore resemblance to three of the six questioned tires, indicating that those tires had not been treated. The remaining three questioned tires, however, were highly suspect. Signal for these samples was an order of magnitude greater than that of the negative control, and the 


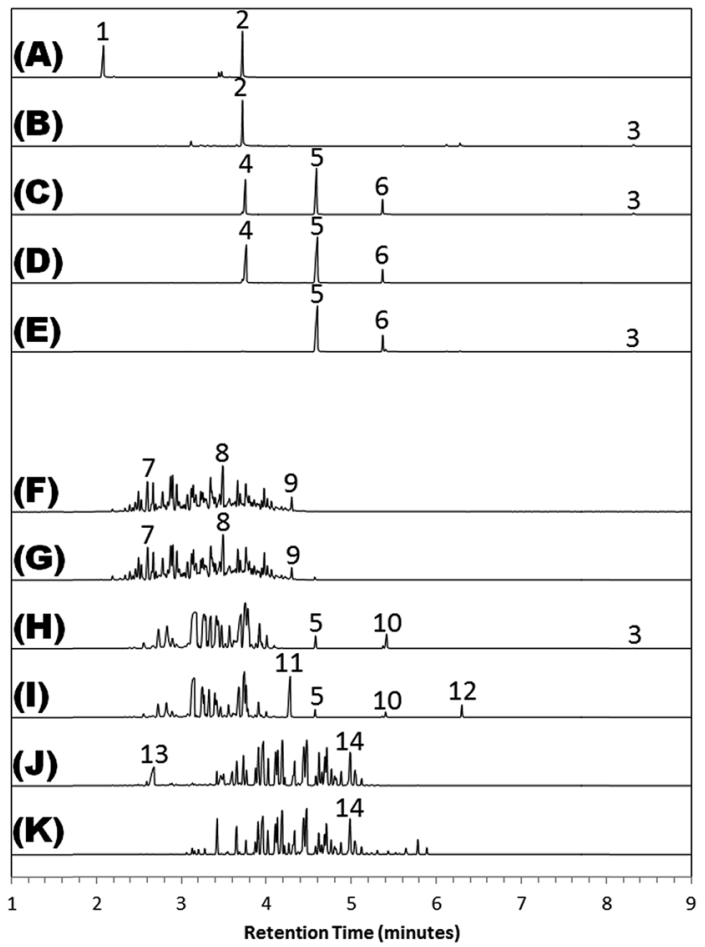

Fig. 1 GC-MS chromatograms for tire treatments (A) Tire Tac Blue Tire Wash, (B) Tire Tac Tire Tuff, (C) Tire Tac GK1, (D) Tire Tac STS5 Purple, (E) Venom Juice Red Hot, (F) Quick Lap Undetectable, (G) Hot Lap Undetectable, (H) TireTac PRW, (I) TireTac SAA, (J) Pro-Blend Motorsports Tire Cleaner, and (K) Tire Tac BGTP Red. The major library hits are (1) 1-propoxy-2-propanol, (2) 2-ethyl-1-hexanol, (3) benzoic acid 2-ethylhexyl ester, (4) butanedioic acid dimethyl ester, (5) pentanedioic acid dimethyl ester, (6) hexanedioic acid dimethyl ester, (7) nonane, (8) decane, (9) undecane, (10) 1-phenoxy-2-propanol, (11) benzoic acid methyl ester, (12) butyl benzoate, (13) 2-butoxyethanol, and (14) naphthalene.

chromatograms were populated with an abundance of nalkanes and cycloalkanes, including nonane, decane, undecane, propylcyclohexane, and butylcyclohexane. Furthermore, all three of the suspect tires were chromatographically indistinguishable from one another. Comparison to known commercial products revealed the tires were likely treated with a product like Tire Treatment F, whose distribution of chromatographic peaks was highly consistent.

Another case study is presented in Fig. 4. Here, the questioned tire displayed a broad envelope of largely aromatic molecules. For the most part, these took the form of mono-, di-, tri-, and tetra-methylated benzene compounds, whose mass spectra were so similar to one another as to impede a conclusive library match. Also identified were the unconventional compounds 2-butoxyethanol and dipropylene glycol monomethyl ether, as well as decane, undecane, and naphthalene. Once again, the chromatogram had little in common with the accompanying negative control that had been submitted, but bore a great deal of resemblance to a known tire treatment.

In this case, Treatment $\mathrm{J}$ was found to contain many of the same compounds as the questioned tire, with a highly-similar distribution of peaks. However, several key differences are of

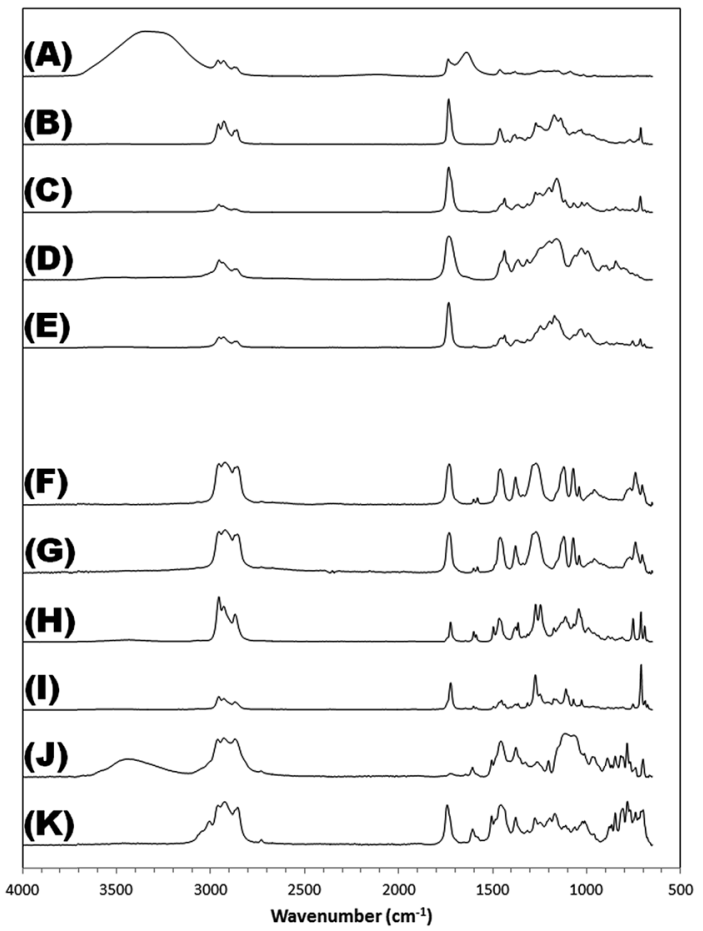

Fig. 2 FTIR spectra for tire treatments. Letter identities the same as in Fig. 1.

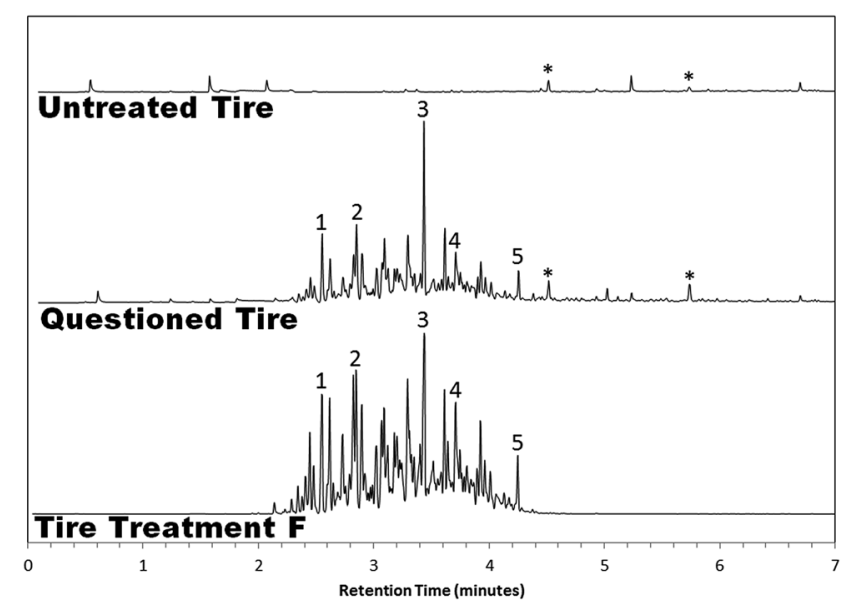

Fig. 3 Chromatographic comparison between an untreated tire, a questioned tire, and a suspect tire treatment. The labeled peaks are (1) nonane, (2) propylcyclohexane, (3) decane, (4) butylcyclohexane, and (5) undecane.

note. Nonane is present in the questioned tire, but is absent in the treatment. Likewise, a trio of high-boiling naphthalenerelated peaks located between 5.6 and 5.9 minutes are nowhere to be found in the chromatogram for the tire treatment product. Perhaps most telling of all, pentanedioic acid dimethyl ester and hexanedioic acid dimethyl ester (but not butanedioic acid dimethyl ester) occurred in the questioned tire chromatogram. The presence of these additional compounds suggests the possibility that a combination of treatment products may have been applied to the tire, with Tire Treatment $\mathrm{J}$ forming the 


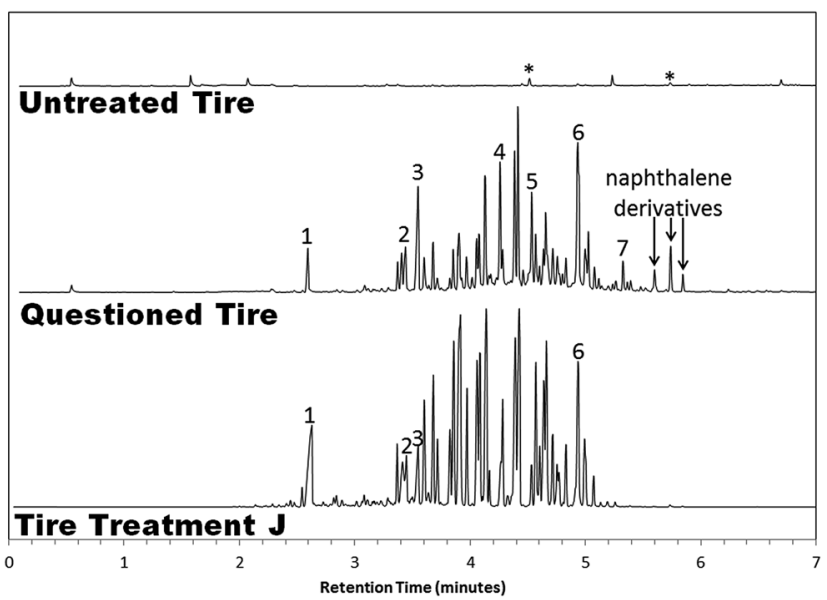

Fig. 4 Chromatographic comparison between an untreated tire, a questioned tire, and a suspect tire treatment. The labeled peaks are (1) 2-butoxyethanol, (2) decane, (3) dipropylene glycol monomethyl ether, (4) undecane, (5) pentanedioic acid dimethyl ester, (6) naphthalene, and (7) hexanedioic acid dimethyl ester.

principal residue left behind, along with small traces of another unknown product. Alternatively, a singular treatment may have been used, highly similar to Tire Treatment $\mathrm{J}$ in chemical composition, but also incorporating nonane and the assorted naphthalene- and plasticizer-related compounds in its formulation.

In cases where a tire treatment is suspected, important information can occasionally be gleaned by evaluating cuttings from the interior and exterior of a questioned tire independent of one another. A longitudinal bisection of the rubber yields two halves, each of which is submitted to analysis by SPME-GC-MS. Fig. 5 portrays the results of such an experiment. It is clear from the data shown that the vast majority of the volatiles originate from the outer surface of the tire, not the inner surface. This type information can prove essential in concluding whether

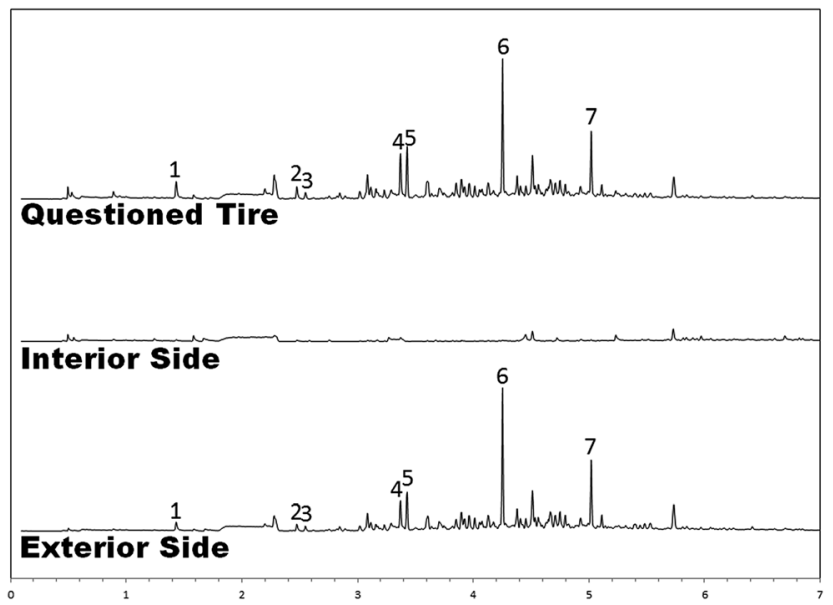

Fig. 5 Chromatographic comparison between a questioned tire and the front and back sides of the same tire. The labeled peaks are (1) toluene, (2) meta and para xylene, (3) ortho xylene, (4) 1,2,4-trimethylbenzene, (5) decane, (6) undecane, and (7) dodecane. a product has been applied: many commercial formulations instruct the end user to coat the interior surface area of the tire with product while leaving the exterior untreated-presumably to avoid detection by race officials, who would not find it difficult to evaluate the easily-accessible outer surface by sight and by touch. A tire sample whose interior surface yields a plethora of volatile compounds in contrast to the exterior, then, is essentially a smoking gun: there is no conceivable way that the sealed interior of any tire could come to be tainted with suspicious volatile material while leaving the exterior unaffected, except by deliberate action. Conversely, in the case shown in Fig. 5, the tire interior is blank, whereas the exterior yields a chromatographic profile resembling a mixture of gasoline and medium petroleum distillate. This type of observation is inherently less conclusive, as it leaves open the possibility of contact cross-contamination between the tire and any illicit residue deposited on the racetrack. However, it may still be of use in pinning down a particular treatment product to a questioned tire: if the front side of a tire produces a chromatogram consistent with a "front-side application" product, then that is one more article of evidence in favor of a treatment product having been used.

Above all else, it is important to minimize the risk of false positives as much as possible. The chance of one car's treated tires spreading their contamination to the wheels of other innocent parties via the racetrack is fully within the realm of possibility. Whenever a tire is evaluated and deemed to be suspicious, it should always be compared to a negative control tire, which has been provided by the race officials or a disinterested third party and raced upon the same track, on the same day as the questioned exemplars.

\section{Conclusion}

In summary, a novel technique was developed for the analysis of volatile chemicals applied to automobile racing tires. This represents an innovative and important contribution, for while race events abound throughout the country and the world, published literature on the detection of illicit tire treatments is not at all widespread. The racing circuits' demands for accountability require sensitive chemical determinations. The method described here offers excellent sensitivity, although overall sample throughput was slow (i.e., each sample consumed $25 \mathrm{~min}$ of instrument time). The development of faster and/or portable methods of analysis would be very useful in this regard.

SPME-GC-MS was found to offer a fast and simple way to evaluate tires for the presence of treatment products, with limits of detection around $200 \mathrm{~nL} \mathrm{~g}^{-1}$. Despite the claims of some commercial entities that their formulations are undetectable, we found the reverse to be true. Products could not only be detected days after they had been applied to the tires, but characterized and discriminated as well. Furthermore, their chromatographic profiles allowed them to be categorized into two broad classes: 'plasticizer-based' tire treatments and 'hydrocarbon-based' tire treatments. Localization of a treatment product to a particular side of a tire is possible by 
bisecting the rubber and submitting each half to independent analysis.

\section{Acknowledgements}

The authors would like to thank Eric Rankine and Kyle McCain of the United States Auto Club for their technical assistance, and for providing all commercial tire treatment products and tire samples evaluated in this study. Financial support for this project was provided by the USAC.

\section{References}

1 C. L. Arthur and J. Pawliszyn, Anal. Chem., 1990, 62, 21452148.

2 Z. Zhang and J. Pawliszyn, Anal. Chem., 1993, 65, 1843-1852.

3 Z. Y. Zhang, M. J. Yang and J. Pawliszyn, Anal. Chem., 1994, 66, A844-A853.

4 M. Llompart, L. Sanchez-Prado, J. Pablo Lamas, C. GarciaJares, E. Roca and T. Dagnac, Chemosphere, 2013, 90, 423431.

5 R. S. Il'yasov, E. G. Mokhnatkina, E. E. Potapov, E. V. Sakharova and V. V. Pyzhonkova, Kauch. Rezina, 2009, 14-18.
6 Fed. Regist., 2002, 67, 45587-45625.

7 M. Gagol, G. Boczkaj, J. Haponiuk and K. Formela, Polym. Degrad. Stab., 2015, 119, 113-120.

8 A. M. Dhabbah, S. S. Al-Jaber, A. H. Al-Ghamdi and A. Aqel, Arabian J. Sci. Eng., 2014, 39, 6749-6756.

9 I. Fettig, S. Krueger, J. H. Deubel, M. Werrel, T. Raspe and C. Piechotta, J. Forensic Sci., 2014, 59, 743-749.

10 M. Joshi, Y. Delgado, P. Guerra, H. Lai and J. R. Almirall, Forensic Sci. Int., 2009, 188, 112-118.

11 N. Lorenzo, T. Wan, R. J. Harper, Y. Hsu, M. Chow, S. Rose and K. Furton, Anal. Bioanal. Chem., 2003, 376, 1212-1224.

12 W. Kranz, K. Kitts, N. Strange, J. Cummins, E. Lotspeich and J. Goodpaster, Forensic Sci. Int., 2014, 236, 157-163.

13 W. D. Kranz, N. A. Strange and J. V. Goodpaster, Anal. Bioanal. Chem., 2014, 406, 7817-7825.

14 W. Kranz, K. Kitts, N. Strange, J. Cummins, E. Lotspeich and J. Goodpaster, Forensic Sci. Int., 2014, 236, 157-163.

15 K. Sakai, M. Kamijima, E. Shibata, H. Ohno and T. Nakajima, J. Environ. Monit., 2006, 8, 1122-1128.

16 G. Wypych, Handbook of Odors in Plastic Materials, William Andrew, 2013. 